\title{
Maintainability Analysis of Underground Mining Equipment Using Genetic Algorithms: Case Studies with an LHD Vehicle
}

\author{
Sihong Peng and Nick Vayenas \\ Laurentian University, 935 Ramsey Lake Road, Sudbury, ON, Canada P3E 2C6 \\ Correspondence should be addressed to Nick Vayenas; nvayenas@laurentian.ca
}

Received 29 August 2013; Accepted 9 December 2013; Published 19 February 2014

Academic Editor: Luis A. Cisternas

Copyright ( 12014 S. Peng and N. Vayenas. This is an open access article distributed under the Creative Commons Attribution License, which permits unrestricted use, distribution, and reproduction in any medium, provided the original work is properly cited.

While increased mine mechanization and automation make considerable contributions to mine productivity, unexpected equipment failures and planned or routine maintenance prohibit the maximum possible utilization of sophisticated mining equipment and require a significant amount of extra capital investment. This paper deals with aspects of maintainability prediction for mining machinery. A PC software called GenRel was developed for this purpose. In GenRel, it is assumed that failures of mining equipment caused by an array of factors follow the biological evolution theory. GenRel then simulates the failure occurrences during a time period of interest using genetic algorithms (GAs) coupled with a number of statistical techniques. A group of case studies focuses on maintainability analysis of a Load Haul Dump (LHD) vehicle with two different time intervals, three months and six months. The data was collected from an underground mine in the Sudbury area in Ontario, Canada. In each prediction case study, a statistical test is carried out to examine the similarity between the predicted data set with the real-life data set in the same time period. The objectives of case studies include an assessment of the applicability of GenRel using real-life data and an investigation of the impacts of data size and chronological sequence on prediction results.

\section{Introduction}

With more and more technological advances made available nowadays, the manufacturing industry is producing various types of equipment worth billions of dollars for use by the mining industry throughout the world. For instance, as per the United States Census Bureau in 2008, American mining equipment manufacturers shipped approximately $\$ 4.0$ billion worth of goods [1], compared to $\$ 2$ billion in 2005 [2]. Within these 2008 figures, underground mining machinery (except parts sold separately) accounted for $\$ 1.0$ billion, or 25 percent. It is worthwhile to mention that portable drilling rigs and parts accounted for $\$ 1.7$ billion or 42.5 percent. In some mines, maintenance cost might take up to 50 to 60 percent of total costs [3, 4]. Today, the global economic recession is forcing mining companies to modernize their operations through increased mechanization and automation. To this end, it is desirable to design and utilize mining equipment systems with better reliability and maintainability both for engineers and mining managers. Particularly in a state of economic recession, mining companies are urged to make the best use of enterprise resources including equipment and maintenance crew while dealing with market volatility and shareholders' expectations of profitability. A number of crucial reasons for improving mining equipment reliability and maintainability are summarized as follows [5]:

(1) to maximize profit;

(2) to reduce the cost of poor reliability/maintainability;

(3) to reduce the performance of mining equipment services in an unplanned manner because of short notice;

(4) to provide more accurate short-term forecasts for equipment operating hours;

(5) to overcome challenges imposed by global competition;

(6) to take advantage of lessons learned from other industrial sectors such as aerospace, defense, and nuclear power generation;

(7) to improve workplace safety. 
Step 1. Initialization

Generate initial population $I$ at random or with prior knowledge

Step 2. Fitness evaluation

Evaluate the fitness for all individuals in $I$

Step 3. Selection

Select a set of promising candidates $C$ from $I$

Step 4. Crossover

Apply crossover to the mating pool $C$ for generating a set of offspring $O$

Step 5. Mutation

Apply mutation to the offspring set $O$ for obtaining its perturbed set $O^{\prime}$

Step 6. Replacement

Replace the current population $I$ with the set of offspring $O^{\prime}$

Step 7. Termination

If the termination criteria are not met, go to Step 2.

Pseudocode 1: Pseudocode of a simple genetic algorithm [13].

\section{Maintainability}

The earliest occurrence of the term maintainability was found in a contract in the development of the Wright brothers' airplane in which it was clearly stated that the aircraft should be "simple to operate and maintain" [6]. Today maintenance costs of sophisticated engineering systems are so high that maintainability draws great attention from scientific researchers to operations managers. For instance, a study [7] shows that American manufacturers spend more than 300 billion U.S. dollars on plant maintenance and operations. Therefore, it is understandable that the main objectives of applying maintainability principles to engineering systems are to reduce projected maintenance costs and time, to use maintainability data to estimate system/equipment availability/unavailability, and to determine labor-hours and other related resources needed to perform the projected maintenance.

A system with better maintainability would inherently provide the benefit of lower maintenance costs, less time to recover with lower breakdown frequency (design for simplicity), less complexity of maintenance tasks, and relatively reduced man-hours [8].

Most maintainability functions use the time to repair as independent variable. It is common to use probabilistic or statistical concepts to define a maintainability function, for example, the probability density function. Let $t$ denote the time. Assuming a repair starts at $t=0$ and completes at time $T$, the maintainability can be mathematically defined [2] as follows:

$$
M(T)=\int_{0}^{T} f_{r}(t) d t
$$

or

$$
M(T)=F_{r} \quad(t \leq T),
$$

where $M(T)$ is the maintainability function, $T$ and $t$ are time, $f_{r}(t)$ is the repair time probability density function, and $F_{r}(t)$ is the repair time cumulative distribution function.

From the above, maintainability is the probability of carrying out a repair within a known time period. In maintainability studies, the variable of interest is time to repair (TTR), whereas, in reliability studies, the variable of interest is time between failures (TBF). As a clarification, reliability is defined as the probability of a product, system, or service to perform its expected task under the specified conditions of use over an intended period of time [10-12].

\section{Genetic Algorithms and Their Applications}

In 1975, Holland wrote a ground-breaking book adaptation in natural and artificial systems in which he described a general framework for understanding the mechanisms behind adaptive behaviors in a number of systems over a broad range of timescales [14]. Later on, a series of further research work [1517] revealed further characteristics and potential applications of genetic algorithms (GAs).

A genetic algorithm imitates a biological evolution process and is often used to seek optimal solution to a practical problem, expressed by the best fitted individual string of values (representing parameters of the practical problem). GAs encode the decision variables (or input parameters) of the underlying problem into strings. Each string, called individual, is a candidate solution. To differentiate good candidate solutions from bad candidate solutions, a fitness function is needed as a measure. A fitness function could be a mathematical expression, or a complex computer simulation, or in terms of subjective human evaluation and guide the evolution of solutions to the problem. Pseudocode 1 shows the pseudocode of a simple genetic algorithm [13].

In each of the procedures, three genetic operators will be implemented in each individual candidate solution: selection, crossover (or recombination), and mutation. In a selection operation, superior individuals will be given a better chance to reproduce the next generation, since the fitter gene has tendency to yield good quality offspring which means a better solution to any problem. In a crossover procedure, the individuals obtained from the selection procedure will exchange and combine partial solutions at a certain crossover probability. In a mutation procedure, a small portion of offspring genes is altered to form the new solution.

Research on GAs has a wide spectra from computer science $[18,19]$ to engineering $[15,20]$ and, more recently, to 


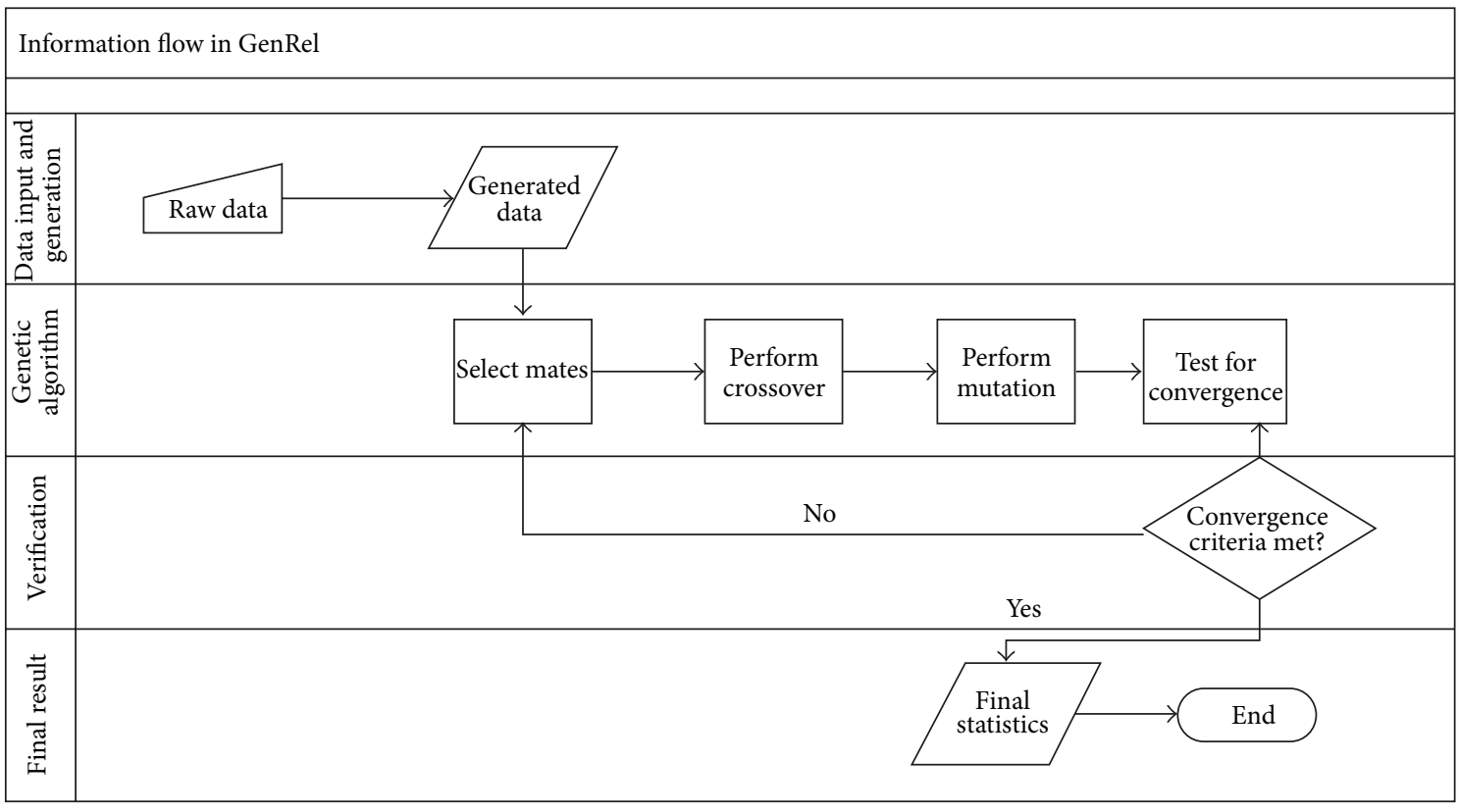

FIgURE 1: A schematic illustration of data flow in GenRel.

fields such as molecular biology, immunology, and economics [21-23]. In the mining engineering field, GAs have been used also for ore grade estimation [24], ore grade optimization [25], solving scheduling and open-pit design problems [26], coal mine production scheduling [27], and open pit equipment selection [28].

Reliability and maintainability models with or without covariates are based on the use of rigorous and complicated statistical techniques which include, for instance, theoretical probability distribution fitting and trend and serial correlation tests and require assumptions of homogeneous or nonhomogeneous Poisson process or assumptions of proportionality of the hazard rate. The assumptions and statistical constraints of probabilistic models limit the ability of these models to accurately represent and fit all real life mining conditions [29]. The authors' research offers an alternative method to the above conventional/statistical methods and provides an approach that can complement the existing maintainability techniques in an effort to contribute to better understanding of failures of capital intensive mining equipment systems over time.

GAs offer several key advantages over conventional mathematical models including simplicity of randomized searches while retaining important historical information with the population, computational simplicity; GAs search from a population of solutions, not just from a single solution, and they can handle any kind of objective function linear or nonlinear constraints defined in discrete, continuous, or mixed search spaces $[15,30]$.

\section{Overview of GenRel}

GenRel is a computer software developed for reliability/ maintainability prediction based on genetic algorithms using historical data. It was developed at Laurentian University Mining Automation Laboratory (LUMAL) and runs in the Microsoft Excel environment.

In a typical genetic algorithm, variables of interest are coded. Afterwards, the processes of mate selection, crossover, and mutation circulate until the fitness function yields desired values [15]. The application of GAs in GenRel can be illustrated in Figure 1.

In GenRel, the user can define the maximum number of iterations, the convergence limit, and the probability of mutation; see Figure 2.

The raw data used in GenRel is derived from historical records in terms of time to repair (TTR). TTR measures the time needed to fix a failure. The distribution fitting process using the software BestFit by Palisade Corporation [31] selects the best fitted probability distribution function $F(x)$ to the raw data. For example, take the exponential distribution [32]

$$
F(x)=1-e^{-(x-\gamma) / \beta},
$$

where $x$ is a TTR value, $\beta$ is the mean, and $\gamma$ is the location parameter. We divide the entire raw data into two equal sets, the raw input data set and the raw evaluation data set. The raw input data set is used to generate new data sets, while the raw evaluation data is used in the evaluation process of the generated data set. Suppose

$$
\begin{array}{ll}
F(x)=1-e^{-\left(x-\gamma_{0}\right) / \beta_{0}}, & x \in\left[\gamma_{0}, \infty\right), \\
\widetilde{F}(x)=1-e^{-\left(x-\widetilde{\gamma}_{0}\right) / \widetilde{\beta}_{0}}, & x \in\left[\widetilde{\gamma}_{0}, \infty\right),
\end{array}
$$

where $\beta_{0}$ and $\widetilde{\beta}_{0}$ are the means, $\gamma_{0}$ and $\widetilde{\gamma}_{0}$ are the location parameters, and $F(x)$ and $\widetilde{F}(x)$ are the best fitted probability distribution functions for the raw evaluation data set and for the raw input data set, respectively. In order to generate new 


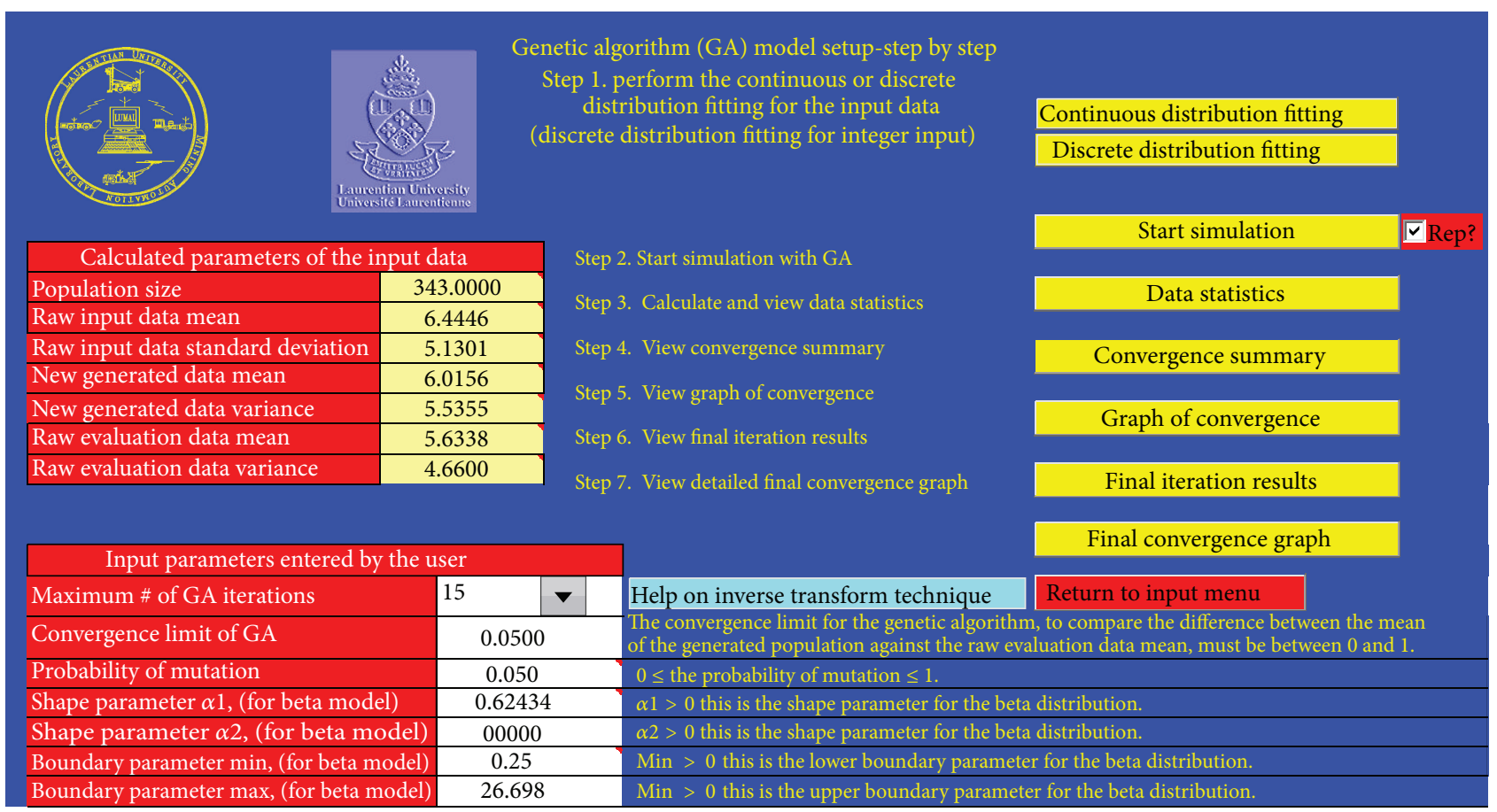

Figure 2: User interface for GenRel to set relevant configurations.

data from the raw input data, we use the inverse transform technique, or ITT [33], by transforming the exponential distribution function into the inverse format and by generating a uniformly distributed random variable $R \sim U(0,1)$. Then, six sets of generated data can be yielded by $x=-\widetilde{\beta}_{0} \ln (1-R)+\widetilde{\gamma}_{0}$ (from (5), an inverse of the function can be expressed as $x=-\widetilde{\beta}_{0} \ln (1-\widetilde{F})+\widetilde{\gamma}_{0}$, where $\widetilde{F} \in[0,1]$; substituting $\widetilde{F}$ with the random variable $R$ yields the expression) After six simulation runs, the created six sets of new data are considered adequate for the prediction process within GenRel.

The generated data is then used for mate selection and crossover in which a random number determines the positions and total number of crossovers, yielding offspring data. Afterwards, mutation is performed at a specified rate defined by the user as mutation probability. Six sets of new offspring data follow the respective best fitted exponential probability distribution functions, denoted by parameter pairs $\left(\beta_{i}, \gamma_{i}\right)$, $(i=1,2,3, \ldots, 6)$, where $\beta_{i}$ and $\gamma_{i}$ represent mean and location of the respective probability distribution functions. A fitness function

$$
f=\frac{\left|\beta_{i}-\beta_{0}\right|+\left|\gamma_{i}-\gamma_{0}\right|}{\left|\beta_{0}\right|+\left|\gamma_{0}\right|} \quad(i=1,2,3, \ldots, 6)
$$

is designed to measure the fitness of each individual offspring data set; for detailed discussion of this procedure, see [34].

If the smallest value of the six fitness function values is not greater than the user-defined convergence limit, then the iterative process is terminated and GenRel is considered applicable for prediction of future data; otherwise, another iteration will be implemented as long as the preset maximum number of iterations is not exceeded and the smallest fitness function value is within a user-defined convergence limit. The convergence limit is the upper limit of deviation between the probability distribution function of the generated data set and the probability distribution function of the raw evaluation data set.

Overall, after the above described algorithmic process is successful, GenRel can be applied to predict future data. Otherwise, GenRel is considered not acceptable for prediction of future data based on the raw data under study. For details of the algorithmic procedure applied in GenRel, see [35]. Besides the exponential probability distribution, GenRel also includes other probability distribution functions (e.g., lognormal, weibull).

\section{Implementation of GenRel}

Implementation of GenRel normally encompasses four major procedures: data preparation, validation of independent and identical distribution (iid) assumption, verification of convergence, and prediction.

5.1. Data Preparation. To fit the data into the maintenance analysis, the original data is processed in the form of time to repair (TTR).

5.2. Trend Test and Serial Correlation Test. Prior to statistical analysis and probability distribution fitting, the data should be tested for trends and serial correlations [3]. The purpose of these tests is to verify the assumption that the data is independent and identically distributed (IID). This step is critical for the probabilistic modeling approach. If the data presents 
a trend or serial correlation, then the data is considered not independent and not identically distributed [33, 36]. In this case, nonstationary maintainability/reliability models are more appropriate than models based on probability distribution fitting to analyze the data.

5.3. Verification of the Applicability of GenRel. If a specific probability distribution function can fit the raw input data, then we can utilize the inverse transform statistical technique [33] to generate new sets of data which conform to the same type of probability distribution function but with slightly different probability distribution function parameter values. In our study, six sets of generated data having the same size as the raw input data set are considered sufficient to mimic the biological process for the prediction of future failures.

To carry out the verification process of GenRel based on historical data, it is necessary to determine the convergence criteria, including the convergence limit, the probability of mutation, and the maximum number of iterations. If the smallest fitness function value of an initially generated data set through the inverse transform statistical technique falls within the convergence limit, then the verification process is considered successful; otherwise, we proceed to the crossover and mutation process, iterating the initially generated data sets, aiming to yield a set of data with acceptable fitness function value, without exceeding the user-defined number of iterations and the probability of mutation. In the case studies proposed in this paper, the following configuration is applied by the end user of GenRel in all verification and prediction processes:

(i) maximum number of GA iterations: 15;

(ii) convergence limit of GA: 0.05;

(iii) probability of mutation: 0.05 .

5.4. Prediction of Data for the Period of Interest. After the successful completion of the verification process for the input data, GenRel then can be applied to predict future data. GenRel is then run to generate the initial offspring data set of the same size as the raw input data set with the parameters values obtained from the raw input data distribution fitting process. In GenRel, six data sets are considered sufficient. In these six data sets, selection, crossover, and mutation are performed until either of the following two criteria is satisfied: (1) the fitness value of the best offspring data set falls within the user-defined convergence limit; (2) total iterations run in GenRel reaches the user-defined maximum number of iterations. Upon completion of the prediction, the $t$-test is used to examine the statistical similarity of the predicted data set with the raw evaluation data set; see [32].

\section{LHD Vehicles in Mining}

A Load Haul Dump vehicle (LHD), also known as a scoop tram, is a specialized loading machine manufactured for the underground mining industry. LHDs are used to scoop extracted ore, with a bucket, load it into the bucket, and dump it in the bottom of the mine to undergo primary crushing before being hoisted to the surface out of the mine. Figure 3 shows the design layout of a typical LHD vehicle.

The Load Haul Dump vehicle concept was introduced to underground mining operations during the late 1960s. With a large bucket in front, the LHD operator sits transversely in the control compartment and drives the LHD in either direction as required. LHD vehicles are designed to perform not only loading but also the hauling function since the haulage distances underground for LHDs are relatively short. They have a large carrying capacity to vehicle size ratio. With the central pin joining design, LHD vehicles can achieve smaller turning radius compared to other similar size vehicles. These features make the LHD a particularly versatile machine. LHDs are mainly diesel powered.

Literature of applications of GenRel to analyze LHD vehicles' reliability can be found in $[35,37,38]$ based on TBF data compiled from historical records. Yet, to the best knowledge of the authors, little has been done with regard to prediction of maintainability of LHD vehicles.

\section{Data Collection and Preprocessing}

Data was gathered from a mine site in Sudbury area in Ontario, Canada, on a daily basis. Operators recorded equipment hours for usage and delays every shift. This information was gathered and reviewed by mine management and organized into a spreadsheet for further analysis [39]. An excerption of the originally obtained data is shown in the left five columns in Table 1.

Since this group of case studies is about the maintainability characteristics of the LHD vehicle, time to repair (TTR) value should be obtained. To this end, several assumptions are made as follows.

(1) If a shift is missing from the data set, then the shift is added and it is assumed that there is no failure during the added shift.

(2) Machine standby time during a shift is registered separately. Table 1 illustrates an example of the final database. The example shows the date, shift code, cumulative time between failures (CTBF), work group, failure code (type of failure), time to repair (TTR), time between failures and machine standby time during a shift.

(3) If a failure code is missing for a repair on a specific shift and it is less than 3 hours, an AV status code is assumed.

The case studies discussed below are based on the collected data sets. Some of the data may not be considered as sufficiently large for statistical analysis (e.g., probability fitting); however, the applicability and usefulness of the proposed methodology can be demonstrated.

\section{Results and Discussion}

8.1. Prediction of TTR Data for Three Months Time Period. Two case studies are considered: prediction of TTR data for the period from April 1 to June 30, 2006 based on historical 


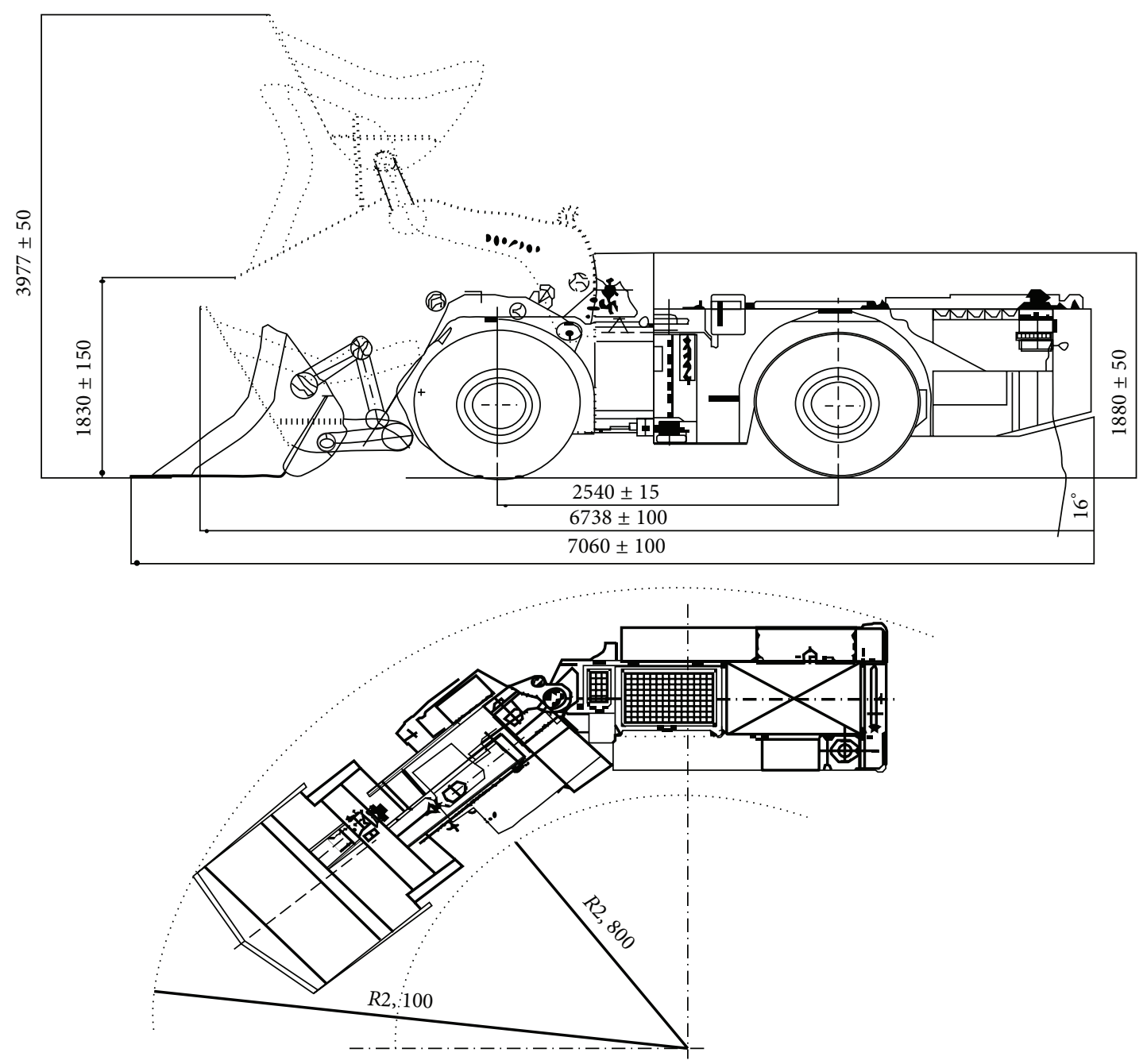

FIgURE 3: This picture presents the schematic design of a typical LHD vehicle commonly used in modern mining industry [9].

TABLE 1: Original LHD vehicle delay records from mine site.

\begin{tabular}{|c|c|c|c|c|c|c|c|}
\hline Date & Shift & CTBF & Group & Failure type & TTR & TBF & Available time \\
\hline 2-Jan-06 & 7 & 8218.6 & Mobile & $\mathrm{MH}$ & 4 & 0.0 & 8.0 \\
\hline 2-Jan-06 & 8 & 8220.0 & Mobile & AV & 0 & 1.4 & 10.6 \\
\hline 3-Jan-06 & 7 & 8228.7 & Mobile & AV & 0 & 8.7 & 3.3 \\
\hline 3-Jan-06 & 8 & 8237.4 & Mobile & AV & 0 & 8.7 & 3.3 \\
\hline 4-Jan-06 & 7 & 8246.1 & Mobile & $\mathrm{AV}$ & 0 & 8.7 & 3.3 \\
\hline 4-Jan-06 & 8 & 8254.8 & Mobile & $\mathrm{AV}$ & 0 & 8.7 & 3.3 \\
\hline 5-Jan-06 & 7 & 8263.4 & Mobile & AV & 0 & 8.7 & 3.3 \\
\hline 5-Jan-06 & 8 & 8263.4 & Mobile & MM & 12 & 0.0 & 0.0 \\
\hline 6-Jan-06 & 7 & 8272.1 & Mobile & $\mathrm{AV}$ & 0 & 8.7 & 3.3 \\
\hline 6-Jan-06 & 8 & 8280.8 & Mobile & $\mathrm{AV}$ & 0 & 8.7 & 3.3 \\
\hline 7-Jan-06 & 1 & 8288.8 & Mobile & AV & 0 & 8.0 & 0.0 \\
\hline 7-Jan-06 & 2 & 8289.5 & Mobile & MM & 2 & 0.7 & 5.3 \\
\hline 7-Jan-06 & 3 & 8289.5 & Mobile & MM & 2 & 0.0 & 6.0 \\
\hline 8-Jan-06 & 7 & 8300.2 & Mobile & AV & 0 & 10.6 & 1.4 \\
\hline 8-Jan-06 & 8 & 8310.8 & Mobile & AV & 0 & 10.6 & 1.4 \\
\hline
\end{tabular}

CTBF: cumulative time between failures; TTR: time to repair; TBF: time between failures. 
TABLE 2: Delay data overview in the periods from January to March 2006 and from October to December 2006.

\begin{tabular}{lcccccc}
\hline $\begin{array}{l}\text { Failure } \\
\text { type }\end{array}$ & \multicolumn{3}{c}{ January-March 2006} & \multicolumn{2}{c}{ October-December 2006} \\
Time & Frequency & $\begin{array}{c}\text { Percent } \\
\text { age (\%) }\end{array}$ & Time & Frequency & $\begin{array}{c}\text { Percent } \\
\text { age (\%) }\end{array}$ \\
\hline AV & 6.3 & 3 & 1.06 & 56.6 & 10 & 14.37 \\
ME & 6 & 2 & 1.01 & 40.7 & 5 & 10.34 \\
MH & 78.1 & 12 & 13.13 & 4 & 1 & 1.02 \\
MM & 389 & 42 & 65.42 & 4.5 & 1 & 1.14 \\
MP & 107.2 & 10 & 18.03 & 208 & 19 & 52.82 \\
OW & 8 & 1 & 1.35 & 80 & 8 & 20.31 \\
\hline Total & 594.6 & 70 & 100 & 393.8 & 44 & 100 \\
\hline
\end{tabular}

AV: available. ME: electrical down. MH: hydraulic down.

MM: mechanical down. MP: planned maintenance. OW: other.

data from January 1, to March 31, 2006, and prediction of TTR data for the period from January 1, to March 31, 2007, based on historical data from October 1, to December 31, 2006. With preliminary manipulations, Table 2 shows an overview of the historical delay data composition in two different time periods, in which AV stands for available, ME for electrical failure, $\mathrm{MH}$ for hydraulic failure, MM for mechanical failure, MP for planned maintenance, and OW for other.

8.1.1. Verification of Applicability of GenRel to the Data Sets under Study. Before GenRel is run, the validity of independent and identical distribution (iid) assumption for the data sets should be checked. Graphical tests show that the data sets under study are independent and identically distributed. Then, the verification process is aimed at providing a prescreening result of GenRel's applicability to the data set under study. In the first case, TTR data for the period of January 1 , to March 31, 2006 is the data set of interest, which is to be used as raw input data set to predict repair data for the period of April 1, to June 30, 2006, if GenRel is to be found applicable through the verification process.

As described in Section 5.4, in the data input interface, TTR data for the period of January to March 2006 is divided chronologically into two parts with equal number of data entries, 35 .

The first step is to find the best fitting distribution function for the raw input data set. The best fitting distribution function, given by @Risk [31], is the normal distribution for the raw input data, with mean $\mu=8.0057$ and standard deviation $\sigma=4.115$.

In the environment of Microsoft Excel with Visual Basic for Applications, a builtin function NORMINV can be directly called to generate random variables from the best fitted normal distribution with location parameter $\mu$ and scale parameter $\sigma$. In light of this convenient ready-to-use function, GenRel produces six sets of data with size identical to that of the raw input data set. These six sets of data generated through the inverse transform statistical technique constitute the initial population for the crossover and mutation operations to follow.
Next, the initial population of six sets of generated data as parents starts to cross over and mutate at a rate of the userdefined mutation probability, 0.05. Elite individuals which possess smaller cost function values have better chance to survive and to be selected to reproduce offspring through selection, crossover, and mutation. These genetic operations iterate until either the maximum number of iterations, which is 15 , or the convergence limit, which is 0.05 , has been reached. In this case, after one iteration, the fitness function value falls within the user-defined convergence limit, as shown in Table 3.

Therefore, it is concluded that GenRel is applicable to analyze the data set from January to March 2006. Similarly and after four iterations GenRel is found applicable to analyze the data set from October to December 2006, as shown in Table 4 .

8.1.2. Prediction of TTR Data for the Time Periods from April to June 2006 and from January to March 2007. After one iteration, GenRel returns a set of TTR data as the prediction of failures on the LHD vehicle during the time period from April to June 2006. Fitting results from @Risk show that the Normal probability distribution fits the predicted data set best. Parameters of the normal probability distribution include $\mu=8.541$ and $\sigma=4.1901$. At a level of significance of $5 \%, t$-test statistic is 1.99 with a degree of freedom of 140 . Based on the result of the $t$-test, it is concluded that there is no significant difference between generated data set and raw evaluation data set in terms of mean at a given level of significance of $5 \%$.

After one iteration in GenRel, a set of TTR data is acquired to project the maintenance characteristic of the LHD vehicle during the period from January to March 2007 based on historical records during the time period from October to December 2006. The software @Risk is used to seek the probability distribution function that best fits the predicted data set. The fitting result shows the normal probability distribution function is the best fit with parameters $\mu=$ 8.9502 and $\sigma=3.5633$. At a level of significance of $5 \%, t$-test statistic is 0.63 with a degree of freedom of 100 . Based on the result of the $t$-test, it is concluded that there is no significant difference between generated data set and raw evaluation data set in terms of mean at a given level of significance of $5 \%$.

The maintainability function of the LHD vehicle during the time periods from April to June 2006 and from January to March 2007 to describe the failure characteristic with respect to repair time can be expressed mathematically in the following equations:

$$
\begin{aligned}
& M(t)=\frac{1}{2}\left[1+\operatorname{erf}\left(\frac{t-8.541}{4.1901 \sqrt{2}}\right)\right], \\
& M(t)=\frac{1}{2}\left[1+\operatorname{erf}\left(\frac{t-8.9502}{3.5633 \sqrt{2}}\right)\right] .
\end{aligned}
$$

Figures 4 and 5 represent maintainability profiles for these two time periods, respectively.

Table 5 shows maintainability function values at some representative TTR. The second row represents maintainability values with respect to TTR from April to June 2006, and 
TABLE 3: Iteration result of TTR data for the period from January to March 2006.

\begin{tabular}{lccccc}
\hline Iteration & Sum of distribution parameters & Upper limit & Lower limit & Deviation of generated data & Accepted convergence? \\
\hline 1 & 12.1207 & 0.606035 & 0 & 0.1452 & Yes \\
\hline
\end{tabular}

TABLE 4: Iteration result of TBF data for the period from October to December 2006.

\begin{tabular}{lccccc}
\hline Iteration & Sum of distribution parameters & Upper limit & Lower limit & Deviation of generated data & Accepted convergence? \\
\hline 1 & 7.97248 & 0.398624 & 0 & 0.40962 & No \\
2 & 7.97248 & 0.398624 & 0 & 0.40962 & No \\
3 & 7.97248 & 0.398624 & 0 & 0.40962 & No \\
4 & 7.97248 & 0.398624 & 0 & 0.05513 & Yes \\
\hline
\end{tabular}

TABLE 5: A table of maintainability function values with representative TTR values.

\begin{tabular}{lccccccc}
\hline TTR (hours) & 3 & 5 & 7 & 9 & 11 & 13 & 15 \\
\hline Maintainability & $0.41 \%$ & $4.55 \%$ & $23.10 \%$ & $58.67 \%$ & $87.97 \%$ & $98.33 \%$ & $99.90 \%$ \\
\hline Maintainability & $0.04 \%$ & $1.33 \%$ & $13.68 \%$ & $51.11 \%$ & $87.50 \%$ & $98.85 \%$ & $99.97 \%$ \\
\hline
\end{tabular}

TABLE 6: Delay data composition for the period from January to June 2006.

\begin{tabular}{lcccccc}
\hline $\begin{array}{l}\text { Type of } \\
\text { delay }\end{array}$ & \multicolumn{3}{c}{ January-June 2006} & \multicolumn{3}{c}{ May-October 2006} \\
Time & Frequency & $\begin{array}{c}\text { Percent } \\
\text { age (\%) }\end{array}$ & Time Frequency & $\begin{array}{c}\text { Percent } \\
\text { age (\%) }\end{array}$ \\
\hline AV & 28.3 & 9 & 2.17 & 102 & 21 & 10.48 \\
ME & 74 & 10 & 5.69 & 44.7 & 7 & 4.59 \\
MH & 294.1 & 32 & 22.60 & 279.6 & 27 & 28.73 \\
MI & 4 & 1 & 0.31 & 4 & 1 & 0.41 \\
MM & 720.9 & 74 & 55.40 & 480.9 & 46 & 49.41 \\
MP & 172 & 16 & 13.22 & 62 & 7 & 6.37 \\
OW & 8 & 1 & 0.61 & 0 & 0 & 0.00 \\
\hline Total & 1301.3 & 143 & 100 & 973.2 & 109 & 100 \\
\hline
\end{tabular}

the third row represents maintainability values with respect to TTR from January to March 2007.

8.2. Prediction of TTR Data for Six Months Time Period. In order to compare with the results obtained for a threemonth period, a group of predictions of TTR data for six months period is carried out. Two case studies are considered: prediction of TTR data for the period from July 1, to December 31, 2006, based on historical data from January 1, to June 30, 2006, and prediction of TTR data for the period from November 1, 2006, to April 30, 2007, based on historical data from May 1 to October 31, 2006. Table 6 shows an overview of the historical delay data composition in two different time periods.

8.2.1. Verification of Applicability of GenRel to the Data Sets under Study. Similar graphical testing processes as aforementioned are implemented and show that the two data sets under study are both independent and identically distributed. Tables 7 and 8 show that after one iteration, GenRel yields an offspring data set within the convergence limit in the two

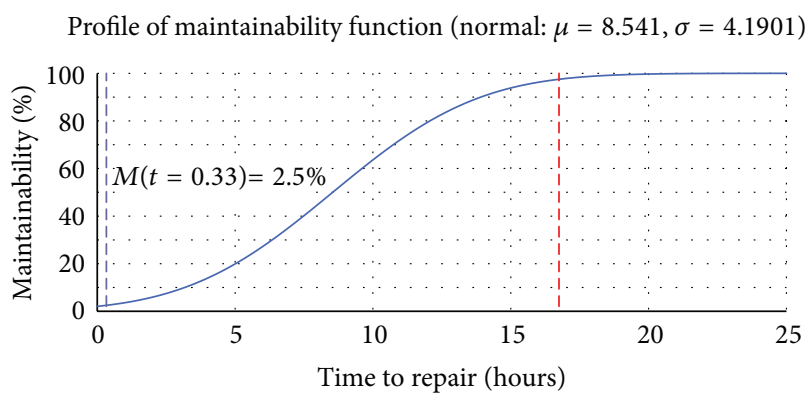

FIgURE 4: The maintainability profile of the LHD vehicle during the time period from April to June 2006.

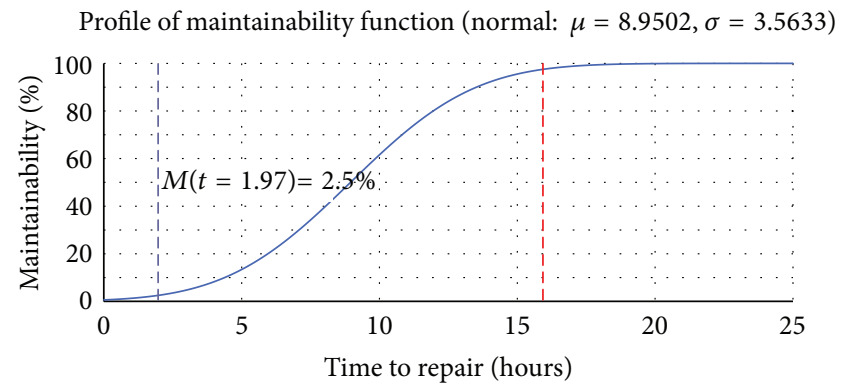

FIGURE 5: The maintainability profile of the LHD vehicle during the time period from January to March 2007.

cases, respectively. Therefore, GenRel is considered applicable to predict future repair data set based on the given historical data sets under this set of configurations.

8.2.2. Prediction of TTR Data for the Time Period from July to December 2006. With one iteration, GenRel renders a set of TTR data to project the maintainability characteristic of the LHD vehicle during the time period from July to December 2006. It is found that this set of TTR data follows the normal probability distribution, according to the fitting result from 
TABLE 7: Iteration result of TTR data for the period from January to June 2006 (verification).

\begin{tabular}{lccccc}
\hline Iteration & Sum of distribution parameters & Upper limit & Lower limit & Deviation of generated data & Accepted convergence? \\
\hline 1 & 12.2817 & 0.614085 & 0 & 0.316 & Yes \\
\hline
\end{tabular}

TABLE 8: Iteration result of TTR data for the period from May to October 2006 (verification).

\begin{tabular}{lccccc}
\hline Iteration & Sum of distribution parameters & Upper limit & Lower limit & Deviation of generated data & Accepted convergence? \\
\hline 1 & 12.9152 & 0.64576 & 0 & 0.1336 & Yes \\
\hline
\end{tabular}

TABLE 9: A table of maintainability function values with representative TTR values.

\begin{tabular}{lccccccc}
\hline TTR (hours) & 3 & 5 & 7 & 9 & 11 & 13 & 15 \\
\hline Maintainability & $0.03 \%$ & $1.13 \%$ & $12.57 \%$ & $49.50 \%$ & $86.91 \%$ & $98.80 \%$ & $99.97 \%$ \\
\hline
\end{tabular}

@Risk. The normal probability distribution is defined with the parameters $\mu=9.0221$ and $\sigma=3.5256$. For the prediction of TTR from November 2006 to April 2007, GenRel also yields a set of data which falls within the user-defined limit. It is found that the best fitting result is the exponential probability distribution defined with the parameters $\beta=7.8884$ and $\gamma=0.88779$.

Thus, the maintainability functions are shown as follows:

$$
\begin{gathered}
M(t)=\frac{1}{2}\left[1+\operatorname{erf}\left(\frac{t-9.0221}{3.5256 \sqrt{2}}\right)\right], \\
M(t)=1-e^{-(t-0.88779) / 7.884} .
\end{gathered}
$$

Figures 6 and 7 reveal maintainability profiles of the LHD vehicle during the time periods from July to December 2006 and from November 2006 to April 2007, respectively. Table 9 gives maintainability function values at representative TTRs.

\section{Conclusion}

In historical TTR data, mechanical failures (MM) consume the most repair time in all the time periods under consideration. The case studies discussed in this paper indicate a successful application of a genetic algorithms based software, GenRel, to predict maintenance characteristics of an LHD vehicle, expressed in terms of maintainability. Two separate groups of case studies at time intervals of three months and six months both present acceptable prediction results at a given level of confidence, $5 \%$. At each time interval, two case studies are investigated and no significant impact of chronological sequence on prediction results is found. Further study is required to investigate how to incorporate the predicted results to improve preventative maintenance policies in practice in underground hard rock mines.

\section{Conflict of Interests}

The authors declare that there is no conflict of interests regarding the publication of this paper.

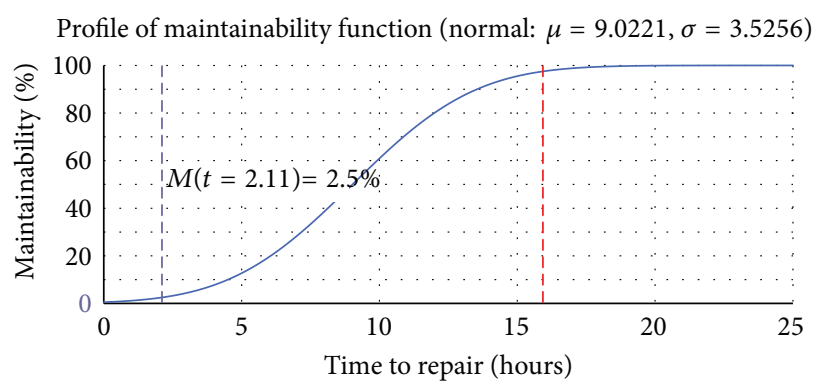

FIGURE 6: The maintainability profile of the LHD vehicle during the time period from July to December 2006.

Profile of maintainability function (exponential: $\beta=7.8884, \gamma=0.88779$ )

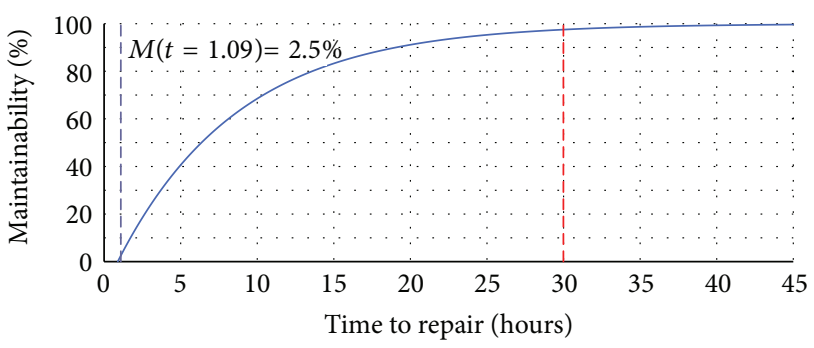

Figure 7: The maintainability profile of the LHD vehicle during the time period from November 2006 to April 2007.

\section{Acknowledgment}

The authors wish to thank the Natural Sciences and Engineering Research Council of Canada (NSERC) for the funding support related to this research.

\section{References}

[1] US Census Bureau, (Current Industrial Reports) Mining Machinery and Mineral Processing Equipment, U.S. Census Bureau, Suitland, Md, USA, 2009.

[2] B. S. Dhillon, Mining Equipment Reliability, Maintainability, and Safety, Springer Series in Reliability Engineering, Springer, New York, NY, USA, 2008. 
[3] N. Vagenas, N. Runciman, and S. R.clément, "A methodology for maintenance analysis of mining equipment," International Journal of Surface Mining, Reclamation and Environment, vol. 11, no. 1, pp. 33-40, 1997.

[4] A. K. S. Jardine and A. H. C. Tsang, Maintenance, Replacement, and Reliability: Theory and Applications, CRC/Taylor \& Francis, Boca Raton, Fla, USA, 2006.

[5] S. Dunn, "Optimising production scheduling for maximum plant utilisation and minimum downtime: the reliability revolution," in Proceedings of the Dollar Driven Mining Conference, Perth, Australia, July 1997.

[6] AMCP706-133, Engineering Design Handbook: Maintainability Engineering Theory and Practice, US Department of Defense, Washington, DC, USA, 1976.

[7] C. J. Latino, "Hidden treasure: eliminating chronic failures can cut maintenance costs up to $60 \%$," Technical Report, Reliability Center, Hopewell, Va, USA, 1999.

[8] AMCP706-134, Engineering Design Handbook: Maintainability Guide for Design, US Department of Defense, Washington, DC, USA, 1972.

[9] "Everpros Mining Machinery Co., Ltd. 2m3 lhd loader," underground mining loader, load haul dump vehicle, lhd vehicle, underground mining vehicle, 2010.

[10] International Electrotechnical Commission, "List of basic terms and definitions and related mathematics for reliability," IEC Publication 271-1974, 1974.

[11] W. J. Layman, "Fundamental consideration in preparing a master system plan," Electric World, vol. 101, pp. 778-792, 1933.

[12] S. A. Smith, "Service reliability measured by probabilities of outage," Electric World, vol. 103, pp. 371-374, 1934.

[13] C. W. Ahn, Advances in Evolutionary Algorithms-Theory, Design and Practice, Volume 18 of Studies in Computational Intelligence, Springer, Berlin, Germany, 2006.

[14] K. F. Man, K. S. Tang, and S. Kwong, Genetic Algorithms, Springer, London, UK, 1999.

[15] D. E. Goldberg, Genetic Algorithms in Search, Optimization, and Machine Learning, Addison-Wesley, Reading, Mass, USA, 1989.

[16] L. D. Davis and M. Mitchell, Handbook of Genetic Algorithms, Van Nostrand Reinhold, New York, NY, USA, 1991.

[17] J. Michalewicz, Genetic Algorithms + Data Structures = Evolution Programs, Springer, Berlin, Germany, 3rd edition, 1999.

[18] L. A. Rendell, "Genetic plans and the probabilistic learning system: synthesis and results," in Proceedings of an International Conference on Genetic Algorithms and Their Applications, pp. 60-73, July 1985.

[19] H. Gunadhi and A. Segev, "A framework for query optimization in temporal databases," in Proceedings of the 5th International Conference on Statistical Scientific Databases, Z. Michalewicz, Ed., vol. 420 of Lecture Notes in Computer Science, pp. 131-147, Springer, New York, NY, USA, 1990.

[20] M. Gen and R. Cheng, Genetic Algorithms \& Engineering Design, John Wiley \& Sons, New York, NY, USA, 1997.

[21] H. Aytug, M. Khouja, and F. E. Vergara, "Use of genetic algorithms to solve production and operations management problems: a review," International Journal of Production Research, vol. 41, no. 17, pp. 3955-4009, 2003.

[22] R. Cheng, M. Gen, and Y. Tsujimura, "A tutorial survey of job-shop scheduling problems using genetic algorithms-I. Representation," Computers and Industrial Engineering, vol. 30, no. 4, pp. 983-997, 1996.
[23] N. C. Proudlove, S. Vaderá, and K. A. H. Kobbacy, "Intelligent management systems in operations: a review," Journal of the Operational Research Society, vol. 49, no. 7, pp. 682-699, 1998.

[24] S. Clement and N. Vagenas, "Use of genetic algorithms in a mining problem," International Journal of Surface Mining, Reclamation \& Environment, vol. 8, no. 4, pp. 131-136, 1994.

[25] M. Ataei and M. Osanloo, "Using a combination of genetic algorithm and the grid search method to determine optimum cutoff grades of multiple metal deposits," International Journal of Surface Mining, Reclamation and Environment, vol. 18, no. 1, pp. 6078, 2004.

[26] B. Denby and D. Schofield, "Open-pit design and scheduling by use of genetic algorithms," Transactions of the Institution of Mining \& Metallurgy A, vol. 103, pp. A21-A26, 1994.

[27] P. C. Pendharkar and J. A. Rodger, "Nonlinear programming and genetic search application for production scheduling in coal mines," Annals of Operations Research, vol. 95, no. 1-4, pp. 251267, 2000.

[28] A. Haidar and S. Naoum, "Selection of opencast mine equipment using knowledge base and genetic algorithm," in Proceedings of the 4th International Symposium on Mine Planning and Equipment Selection, R. K. Singhal, Ed., pp. 409-414, Taylor \& Francis, Calgary, Canada, November 1995.

[29] N. Vagenas and T. Nuziale, "Genetic algorithms for reliability assessment of mining equipment," Journal of Quality in Maintenance Engineering, vol. 7, no. 4, pp. 302-311, 2001.

[30] R. L. Haupt and S. E. Haupt, Practical Genetic Algorithms, John Wiley and Sons, New Jersey, NJ, USA, 2nd edition, 2004.

[31] Palisade Corporation, Bestfit Distribution Fitting, Palisade Corporation, Ithaca, NY, USA, 2010.

[32] G. K. Kanji, 100 Statistical Tests, SAGE, Thousand Oaks, Calif, USA, 2006.

[33] A. M. Law and W. D. Kelton, Simulation Modeling and Analysis, McGraw-Hill, Boston, Mass, USA, 2000.

[34] X. Wu, Reliability assessment of mobile mining equipment using genetic algorithms combined with maintenance analysis: a case study of a fleet of load-haul-dump (lhd) vehicles [M.S. thesis], Laurentian University, Ontario, Canada, 2009.

[35] N. Vayenas and X. Wu, "Genrel: a computerised model for reliability prediction of mining machinery," International Journal of Mining and Mineral Engineering, vol. 1, no. 4, pp. 365-382, 2009.

[36] L. M. Leemis, Reliability: Probabilistic Models and Statistical Methods, Prentice-Hall, Englewood Cliffs, NJ, USA, 1995.

[37] T. Nuziale and N. Vagenas, "A software architecture for reliability analysis of mining equipment," International Journal of Surface Mining, Reclamation and Environment, vol. 14, no. 1, pp. 19-34, 2000.

[38] G. Yuriy and N. Vayenas, "Discrete-event simulation of mine equipment systems combined with a reliability assessment model based on genetic algorithms," International Journal of Mining, Reclamation and Environment, vol. 22, no. 1, pp. 70-83, 2008.

[39] S. Peng, Application of GenRel for reliability and maintainability analysis of underground mining equipment: three case studies [M.S. thesis], Laurentian University, Sudbury, Ontario, 2011. 

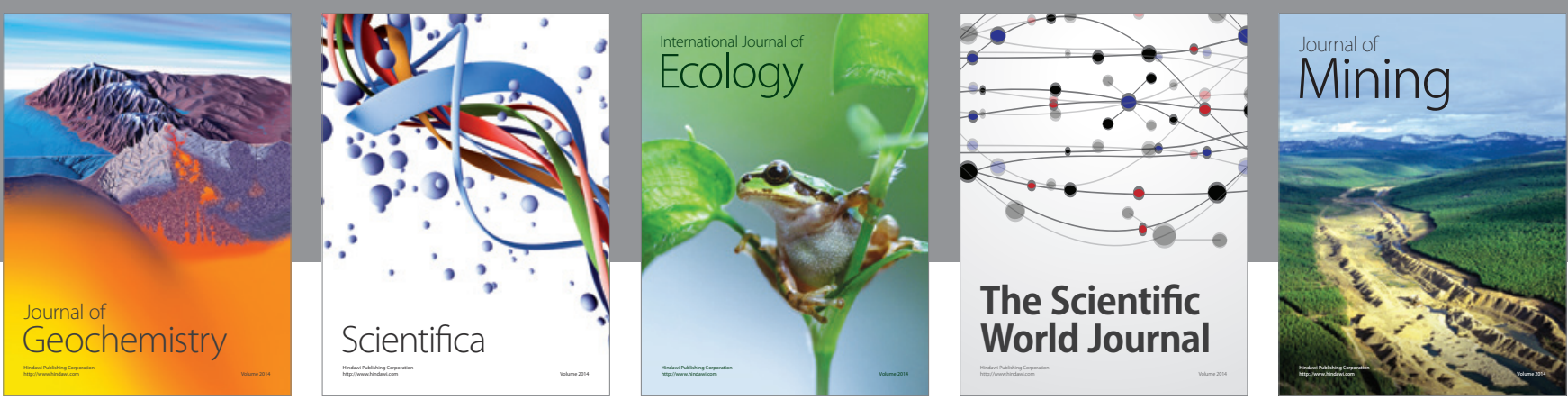

The Scientific World Journal
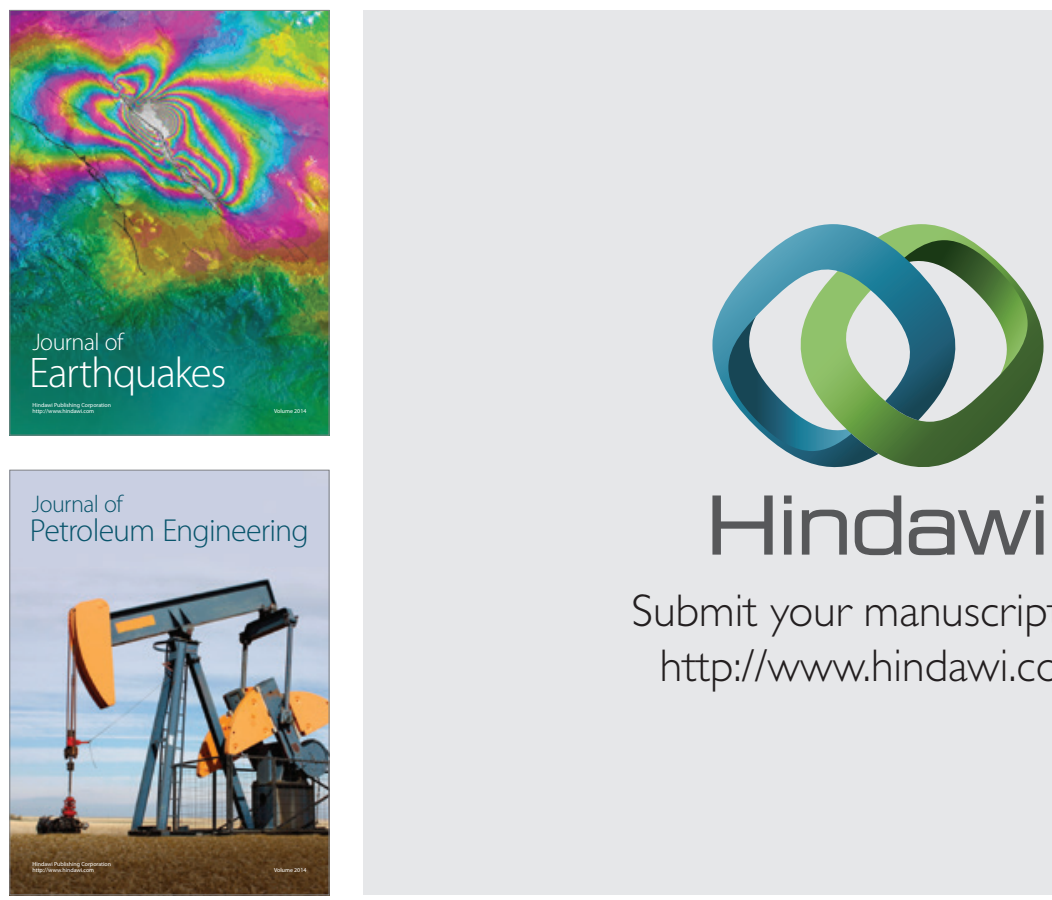

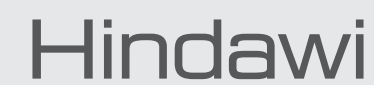

Submit your manuscripts at

http://www.hindawi.com
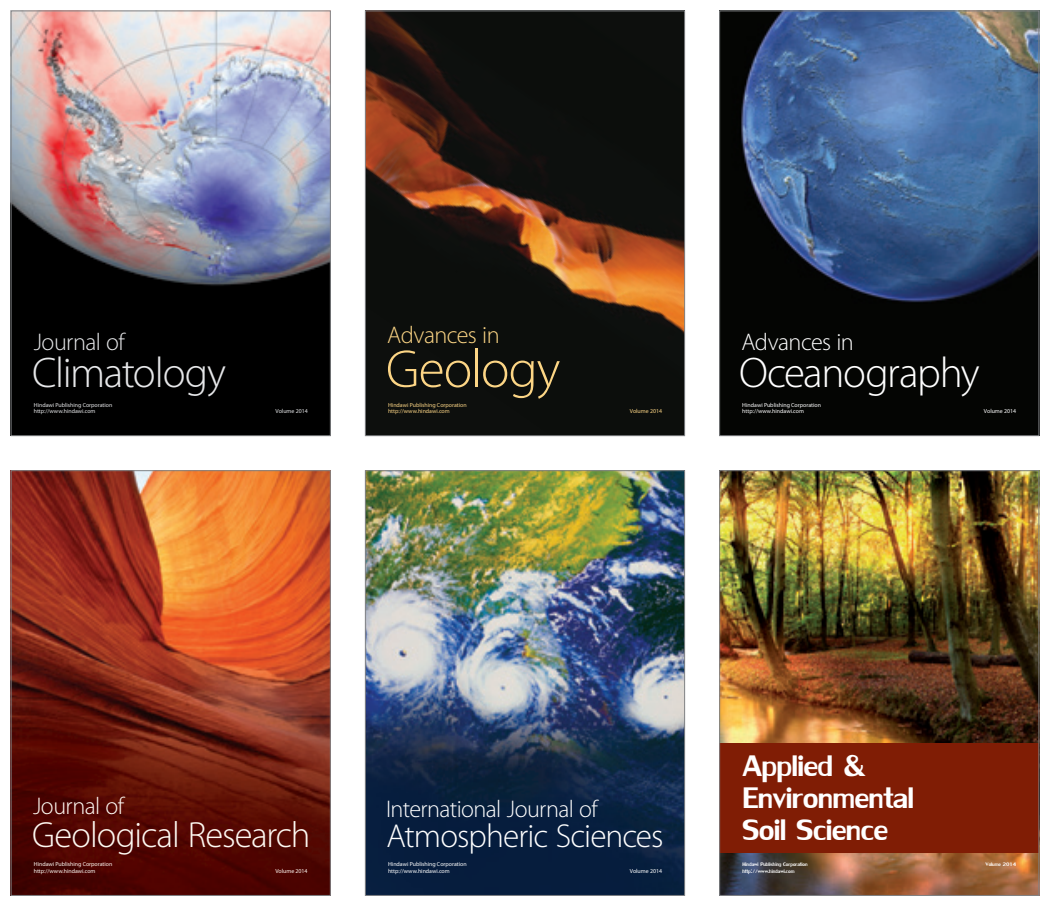
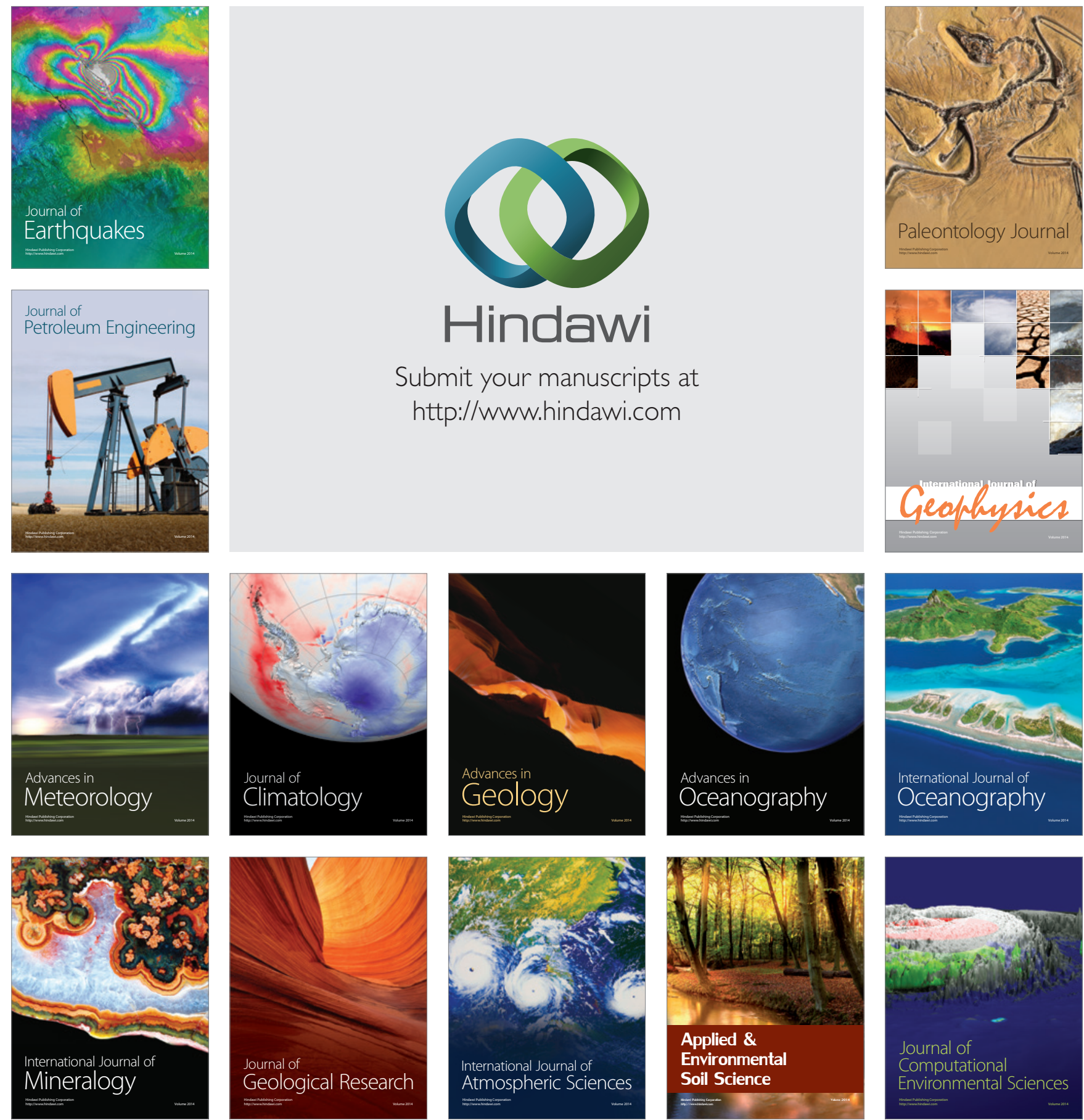